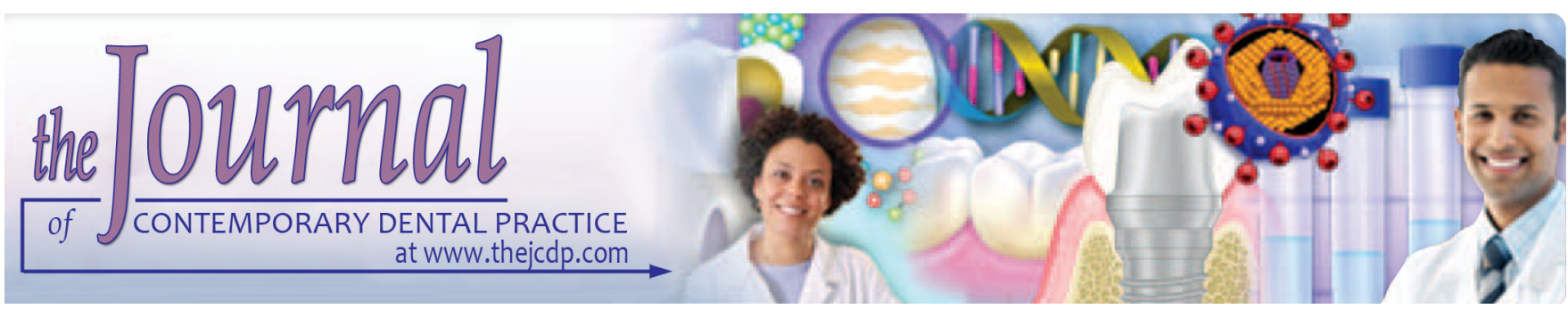

\title{
Oral Health Knowledge and Attitude among Primary School Teachers of Madinah, Saudi Arabia
}

\author{
Mohammad Sami Ahmad
}

\begin{abstract}
Background and objective: Effect of oral health knowledge and attitude has direct effect on school children so aim of this study is to know the oral health knowledge and attitude among primary school teachers.
\end{abstract}

Materials and methods: This was a cross-sectional questionnaire based survey done in 2014. Four males and three females schools were selected using a convenience sampling method. Among them, three were private and the rest was government schools. Approval was obtained from the selected schools. One hundred twenty self-administered questionnaires in Arabic language were distributed among the primary school teachers.

Results: Completed questionnaires were returned with a response rate of $95 \%$. Among the teachers, $57 \%$ were males and rests were females. The mean age was 36.1 years (SD \pm 6.9 ). Sixty-eight percent were between 31 and 40 years old and among them female showed high score for oral health knowledge $(80 \%) p<0.001$ whereas male showed high score of attitude $(82 \%) p<0.05$. Thirty-three percent had 5 and 10 years of teaching experience. Ninety-eight percent were graduate or above. Eighty-nine percent had used toothbrush. There was no significant relation between the teaching experience and the oral health knowledge $(p=0.14)$ but there was a significant relation between teaching experience and attitude $(p=0.001)$. In this sample, irrespective of their frequency of tooth brushing, a significant number had good knowledge $(p<0.001)$ and highly acceptable attitudes $(p=0.001)$ toward oral health.

Conclusion: Primary school teachers had acceptable knowledge and attitudes regarding their oral health. Further studies are needed to evaluate and compare their oral health status to their knowledge and attitudes and to determine whether they offer oral health education to the school children.

Department of Preventive Dental Sciences, College of Dentistry, Taibah University, Madinah, Saudi Arabia

Corresponding Author: Mohammad Sami Ahmad, Associate Professor in Dental Public Health, Department of Preventive Dental Sciences, PO Box 2898, Madinah 43353, Saudi Arabia Phone: 0531640794 e-mail: msamiahmad@yahoo.com
Keywords: Arabic, Attitude, Oral health, Primary school.

How to cite this article: Ahmad MS. Oral Health Knowledge and Attitude among Primary School Teachers of Madinah, Saudi Arabia. J Contemp Dent Pract 2015;16(4):275-279.

\section{Source of support: Nil}

\section{Conflict of interest: None}

\section{INTRODUCTION}

Most common dental diseases like dental caries and periodontal diseases are preventable. ${ }^{1}$ Majority of the school children worldwide and most adults have an experience of dental caries. ${ }^{2}$ A meta-analysis study revealed that there was a high prevalence of dental caries among both adults and children in Saudi Arabia. ${ }^{3}$ To prevent dental caries and other dental diseases, it is important to improve the oral hygiene and provide oral health knowledge to the community. It is important to educate communities about the etiology and prevention of oral diseases. The ideal group to target for the implantation of this knowledge is in the primary school children aged between 6 and 12 years old. This age group is important because at this age both behavioral and attitudinal changes toward dental and general health occur. ${ }^{4}$ Health professionals often target this group by providing oral health checkups and offering oral health education to them. But due to time and budget resources, health professionals cannot visit the schools regularly and consistently which often makes it impossible to change the attitude toward dental health among the children. It needs continuous education to develop positive attitudes. ${ }^{5}$

One option is to allow teachers to introduce and sustain oral health programs at schools. The number of teachers worldwide at the primary school level has been reported to be around 23.9 million. ${ }^{6}$ Teachers are an important and influential part of the society and play an important role in the formation and modification of 
behaviors of young children. Therefore, their knowledge about oral health is important for both their own oral health and the children that they are interacting with and teaching.

School being the first level of learning, may be the most essential aspect in an individual's life. In this regard, World Health Organization (WHO) in 1995 launched a Global School Health Initiative ${ }^{7}$ in which the importance of schools in the delivery of health education to the school children was highlighted. The schools can provide an effective forum to enhance general as well as the oral health awareness among children. ${ }^{8}$ Teachers, with their educational experiences and regular contact with the school children can have positive role in contributing good oral health to the students by promoting oral hygiene. ${ }^{9}$ Knowledge of oral diseases and more importantly about the fact that most of these diseases are preventable to a large extent is a major vehicle for improving the oral health of the children. School teachers are capable of positively influencing and changing the behavior of school children by influencing them with their knowledge. ${ }^{10}$ Documented evidence shows that teachers and parents can augment dental health behavior. ${ }^{11}$ Healthy teeth can last a life time with the proper implementation of dental and oral care. This is possible only when the teachers and children understand the importance of oral health knowledge, attitude, behavior and its practice. ${ }^{12}$ Studies in Saudi Arabia have reported that teacher's positive attitude toward school based dental health education and a willingness to be involved in oral health promotion. ${ }^{13}$ Therefore, it is essential to determine the oral health knowledge and practice among school teachers.

This study was the first in Madinah to evaluate the knowledge and attitudes of primary school teacher's regarding oral health. The results will help in planning workshop or training courses for teachers to improve their knowledge.

\section{MATERIALS AND METHODS}

This was a cross-sectional questionnaire based survey done in 2014. It assessed the knowledge, attitude and practice related to oral hygiene and dental diseases in primary school teachers from Madinah, Saudi Arabia. Most of the schools in Saudi Arabia are run by the government but there are also many privately run schools. Out of a total of thirty males and females primary schools in Madinah, four males and three females schools were chosen using a convenience sampling method. Among them, three were private and the rest was government schools. Before the start of the study, approval was obtained from the selected schools and letters were sent to the schools explaining the purpose of the study and procedures that will be fulfilled during the study. The questionnaire was self-administered in the Arabic language and designed to obtain the oral health knowledge and attitudes of primary school teachers. A total of 120 questionnaires were distributed among the primary school teachers. The questionnaire consisted of three parts; oral hygiene practice, knowledge and attitude toward oral health. The results from the practice section were analyzed individually. The results from the knowledge and attitude sections were allocated a score of either one or zero. The correct answers were given one point and the incorrect answers had a zero point. The total scores were added and oral health knowledge had eight questions with a maximum score of 8 and minimum of zero. The scores were categorized into three levels; poor (0 to 1), average (2 to 4 ) and good (5 to 8 ). To measure the attitude, the same criteria was utilized and the scores were classified as follows; unacceptable (0 to 1), acceptable (2 to 4 ) and highly acceptable (5 to 8 ). The data were analyzed using statistical package for social sciences (SPSS) version 21 and p-value is measured by Chi-square.

\section{RESULTS}

Out of 120 questionnaires, 114 completed questionnaires were returned with a response rate of $95 \%$. All of the questionnaires were fully completed and were included in the analyses. Among all the teachers $57 \%$ were male and $43 \%$ female. The age of the teachers were divided into four groups, 20 to 30, 31 to 40,41 to 50 years and over 51 years for statistical purposes. The mean age was 36.1 years $(\mathrm{SD} \pm 6.9)$. More than half of the school teachers $(62 \%)$ were between 31 and 40 years old and (5\%) were older than 51 years. Less than half (33\%) had between 5 and 10 years of teaching experience and $17 \%$ had more than 15 years of experience. Most of them (98\%) were qualified as a graduate or above. Thirty-nine percent had income less than Saudi Riyal 5000/- and only 5\% had income more than Saudi Riyal 15000/-. Fifty-two percent of teachers were working in a government school whereas $48 \%$ were in private schools (Table 1). The majority $(89 \%)$ claimed to use a toothbrush and $85 \%$ reported using toothpaste to clean their teeth. Only $5 \%$ said that they did not clean their teeth regularly. A significantly higher $(\mathrm{p}<0.001)$ number of females $(80 \%)$ had good oral health knowledge compared to males (52\%) while significantly more males $(p<0.05)$ had good attitude scores $(82 \%)$ compared to females $(61 \%)$ as shown in Table 2. Most of those (68\%) between 31 and 40 years showed high scores for oral health knowledge while for the oral health attitudes, all age groups showed similar results (Table 3). There 
Table 1: Distribution of school teachers according to sex, age, teaching experience, qualification, income and type of school

\begin{tabular}{lll}
\hline Sex & Number & Percentage (\%) \\
\hline Male & 65 & 57 \\
Female & 49 & 43 \\
Age & & \\
21-30 years & 24 & 21 \\
31-40 years & 71 & 62 \\
41-50 years & 14 & 12 \\
More than 50 years & 5 & 5 \\
Teaching experience & & \\
Less than 5 years & 29 & 25 \\
5-10 years & 38 & 33 \\
11-15 years 28 & 28 & 25 \\
More than 15 years & 19 & 17 \\
Qualifications & & \\
Diploma & 2 & 2 \\
Graduate and above & 112 & 98 \\
Income & & \\
Less than SR 5000 & 44 & 39 \\
SR 5001-10000 & 39 & 34 \\
SR 10001-15000 & 25 & 22 \\
More than SR 15000 & 6 & 5 \\
Type of school & & \\
Government school & 59 & 52 \\
Private school & 55 & 48 \\
\hline
\end{tabular}

was no significant relationship between the age and the knowledge or attitude toward oral health. There was no association between the teaching experience and the oral health knowledge or oral health attitudes with more than $50 \%$ having good oral health knowledge and more than $75 \%$ having a highly acceptable attitude toward oral health. There was no significant relation between the teaching experience and the oral health knowledge $(p=0.14)$ but there was a significant relation between teaching experience and attitude $(p=0.001)$ as shown in Table 4. In this sample, irrespective of their frequency of tooth brushing, a significant number had good knowledge $(\mathrm{p}<0.001)$ and highly acceptable attitudes $(\mathrm{p}=0.001)$ toward oral health (Table 5).

\section{DISCUSSION}

The teachers play major role in developing and building character among school children by transmitting their knowledge. There is a national healthcare proposal in Saudi Arabia, to provide and train school teachers regarding how to prevent dental diseases. Most of the schools have one physician and dentist employed from ministry of education who take care of the general and oral health of school children and provide oral health knowledge.

Table 2: Gender distribution of school teacher according to oral health knowledge and attitude

\begin{tabular}{|c|c|c|c|c|c|c|c|c|c|c|c|c|}
\hline \multirow[b]{3}{*}{ Gender } & \multicolumn{6}{|c|}{ Oral health knowledge $p<0.001$} & \multicolumn{6}{|c|}{ Oral health attitude $p<0.05$} \\
\hline & \multicolumn{2}{|c|}{ Poor } & \multicolumn{2}{|c|}{ Average } & \multicolumn{2}{|c|}{ Good } & \multicolumn{2}{|c|}{ Poor } & \multicolumn{2}{|c|}{ Average } & \multicolumn{2}{|c|}{ Good } \\
\hline & $N$ & $\%$ & $N$ & $\%$ & $N$ & $\%$ & $N$ & $\%$ & $N$ & $\%$ & $N$ & $\%$ \\
\hline Male & 2 & 3 & 29 & 45 & 34 & 52 & 2 & 3 & 10 & 15 & 53 & 82 \\
\hline Female & 5 & 10 & 5 & 10 & 39 & 80 & 5 & 10 & 14 & 29 & 30 & 61 \\
\hline Total & 7 & 6 & 34 & 30 & 73 & 64 & 7 & 6 & 24 & 21 & 83 & 73 \\
\hline
\end{tabular}

Table 3: Age distribution of school teacher according to oral health knowledge and attitude

\begin{tabular}{|c|c|c|c|c|c|c|c|c|c|c|c|c|}
\hline \multirow[b]{3}{*}{ Age groups } & \multicolumn{6}{|c|}{$\begin{array}{l}\text { Oral health knowledge } \\
\qquad p=0.36\end{array}$} & \multicolumn{6}{|c|}{$\begin{array}{l}\text { Oral health attitude } \\
\qquad p=0.39\end{array}$} \\
\hline & \multicolumn{2}{|c|}{ Poor } & \multicolumn{2}{|c|}{ Average } & \multicolumn{2}{|c|}{ Good } & \multicolumn{2}{|c|}{ Poor } & \multicolumn{2}{|c|}{ Average } & \multicolumn{2}{|c|}{ Good } \\
\hline & $N$ & $\%$ & $N$ & $\%$ & $N$ & $\%$ & $N$ & $\%$ & $N$ & $\%$ & $N$ & $\%$ \\
\hline $20-30$ years & 0 & 0 & 9 & 38 & 15 & 62 & 0 & 0 & 8 & 33 & 16 & 67 \\
\hline $31-40$ years & 5 & 7 & 18 & 25 & 48 & 68 & 6 & 8 & 14 & 20 & 51 & 72 \\
\hline $41-50$ years & 2 & 14 & 4 & 29 & 8 & 57 & 1 & 7 & 2 & 14 & 11 & 79 \\
\hline$>50$ years & 0 & 0 & 3 & 60 & 2 & 40 & 0 & 0 & 0 & 0 & 5 & 100 \\
\hline Total & 7 & 6 & 34 & 30 & 73 & 64 & 7 & 6 & 24 & 21 & 83 & 73 \\
\hline
\end{tabular}

Table 4: Distribution of experience of school teacher according to oral health knowledge and attitude

\begin{tabular}{|c|c|c|c|c|c|c|c|c|c|c|c|c|}
\hline \multirow{3}{*}{$\begin{array}{l}\text { Teaching } \\
\text { experience }\end{array}$} & \multicolumn{6}{|c|}{$\begin{array}{l}\text { Oral health knowledge } \\
\qquad p=0.14\end{array}$} & \multicolumn{6}{|c|}{$\begin{array}{l}\text { Oral health attitude } \\
\qquad p=0.001\end{array}$} \\
\hline & \multicolumn{2}{|c|}{ Poor } & \multicolumn{2}{|c|}{ Average } & \multicolumn{2}{|c|}{ Good } & \multicolumn{2}{|c|}{ Poor } & \multicolumn{2}{|c|}{ Average } & \multicolumn{2}{|c|}{ Good } \\
\hline & $N$ & $\%$ & $N$ & $\%$ & $N$ & $\%$ & $N$ & $\%$ & $N$ & $\%$ & $N$ & $\%$ \\
\hline$<5$ years & 5 & 17 & 7 & 24 & 17 & 59 & 5 & 17 & 3 & 10 & 21 & 73 \\
\hline $5-10$ years & 2 & 5 & 11 & 29 & 25 & 66 & 1 & 3 & 5 & 13 & 32 & 84 \\
\hline $10-15$ years & 0 & 0 & 9 & 32 & 19 & 68 & 1 & 3 & 13 & 46 & 14 & 50 \\
\hline$>15$ years & 0 & 0 & 7 & 37 & 12 & 63 & 0 & 0 & 3 & 16 & 16 & 84 \\
\hline Total & 7 & 6 & 34 & 30 & 73 & 64 & 7 & 6 & 24 & 21 & 83 & 73 \\
\hline
\end{tabular}


Table 5: Distribution of frequency of tooth brushing of school teacher according to oral health knowledge and attitude

\begin{tabular}{|c|c|c|c|c|c|c|c|c|c|c|c|c|}
\hline \multirow{3}{*}{$\begin{array}{l}\text { Times of } \\
\text { cleaning teeth }\end{array}$} & \multicolumn{6}{|c|}{$\begin{array}{l}\text { Oral health knowledge } \\
\qquad p<0.001\end{array}$} & \multicolumn{6}{|c|}{$\begin{array}{l}\text { Oral health attitude } \\
\qquad p=0.001\end{array}$} \\
\hline & \multicolumn{2}{|c|}{ Poor } & \multicolumn{2}{|c|}{ Average } & \multicolumn{2}{|c|}{ Good } & \multicolumn{2}{|c|}{ Poor } & \multicolumn{2}{|c|}{ Average } & \multicolumn{2}{|c|}{ Good } \\
\hline & $N$ & $\%$ & $N$ & $\%$ & $N$ & $\%$ & $N$ & $\%$ & $N$ & $\%$ & $N$ & $\%$ \\
\hline One time & 0 & 0 & 10 & 32 & 21 & 68 & 0 & 0 & 5 & 16 & 26 & 84 \\
\hline Two times & 0 & 0 & 13 & 35 & 24 & 65 & 1 & 3 & 2 & 5 & 34 & 92 \\
\hline$>$ Two time & 7 & 17 & 7 & 17 & 28 & 66 & 6 & 14 & 16 & 38 & 20 & 48 \\
\hline $\begin{array}{l}\text { Not clean every } \\
\text { day }\end{array}$ & 0 & 0 & 4 & 100 & 0 & 0 & 0 & 0 & 1 & 25 & 3 & 75 \\
\hline Total & 7 & 6 & 34 & 30 & 73 & 64 & 7 & 6 & 24 & 21 & 83 & 73 \\
\hline
\end{tabular}

The response rate was relatively high since the principal investigator went repeatedly to the schools to collect the data. Almost two-thirds were between 31 and 40 years old and this showed that the teachers were a mix of both young and older experienced staff. This balance combines the experience of older teachers with the innovation and youth of younger ones which helps to develop school children and expose them to different cultures and values. Their age also represents their teaching experience as $33 \%$ of them had between 10 and 15 years of experience.

In this study, $68 \%$ of the subjects were cleaning mouth both morning and evening. The prevalence of tooth brushing and using of toothpaste was similar to other studies. ${ }^{5,9,14}$ Females had better knowledge and this is similar to other studies. ${ }^{5}$

It was surprising, however, that males had better attitudes toward dental health compared to females. Females reported more knowledge but perhaps after implementing oral health practices they did not see any benefit or improvement and hence their attitudes changed. The males had poor knowledge but continued to have strong attitudes due perhaps to their culture or upbringing.

The age had no effect on their knowledge and attitudes and this could be due to a lack of continuous school oral health programs that could improve their knowledge. The result of this study is similar to the study of Pradeep et al. ${ }^{15}$ It is, therefore, recommended to offer oral health workshops to teachers in order to improve their oral health knowledge and attitudes.

All of the teachers, irrespective of their experience, had highly acceptable scores for attitudes toward oral health. This showed that although some did not have good knowledge, they still had positive attitudes regarding their oral health. In a previous study, authors have found similar finding among the teachers of English medium school of Telangana, India. ${ }^{16}$

Irrespective of their frequency of tooth brushing, a significant number had good knowledge $(p<0.001)$ and highly acceptable attitudes $(\mathrm{p}=0.001)$ toward oral health. Most of the people had at least five times Miswak practice in Saudi Arabia. This might be reason that they have good knowledge and attitude toward oral health irrespective of frequency of tooth brushing.

\section{CONCLUSION}

This group of primary school teachers had acceptable knowledge and attitudes regarding their oral health but further workshops are recommended to improve their existing knowledge. It is also recommended to carry out further studies to evaluate and compare their oral health status to their knowledge and attitudes and to determine whether they offer oral health education to the school children.

\section{ACKNOWLEDGMENT}

The author would like to thank Dr Ahmed Bhayat for advising him during statistical analysis and would also like to thank Dr Hala Bakeer and Dr May Gamer for helping in filling the questionnaire.

\section{REFERENCES}

1. Schoen MH, Freed JR. Prevention of dental caries: caries and periodontal disease. Annu Rev Public Health 1981;2:71-92.

2. The World Oral Health Report 2003. Continuous improvement in the oral health in the 21st century-the approach of the WHO Global Oral Health Program.

3. Khan SQ, Khan NB, Rejaie ASA. Dental caries: a metaanalysis on a Saudi population. Saud Med J 2013;34(7):744-749.

4. Dawani N, Afaq A, Bilal S. Oral health knowledge, attitude and practices amongst teachers of public school set-up of Karachi, Pakistan. J Dow Uni Health Sci 2013;7(1):15-19.

5. Amith HV, Cruz D, Audrey M, Shirahatti, Ravi V. Knowledge, attitude and practice regarding oral health among the rural government primary school teachers of Mangalore, India. J Dent Hygiene 2013;87(6):362-364.

6. World Education Report. Paris: UNESCO; 1993.

7. Global School Health Initiative. World Health Organization (WHO); 1995. Available at: http://www.who.int/school_ youth_health/gshi/en/Accessed November 2011.

8. The status of school health. Report of the school, health working group and $\mathrm{WHO}$ expert committee on comprehensive school health education and promotion. Geneva: WHO;1996.

9. Manjunath G, Kumar NN. Oral health knowledge, attitude and practices among school teachers in Kurnool, Andhra Pradesh. JOHCD 2013;7(1):17-23. 
10. Harold D, et al. Dental knowledge and attitudes among Arab school teachers in Northern Israel. Int Dent J 1999;49: 269-274.

11. Thomas S, Tandon S, Nair. Effect of dental health education on the oral health status of a rural child population by involving target groups. S J Ind Soc Pedod Prev Dent 2000;18(3): 115-125.

12. Kolawaola KA, Ozieoglie E, Bamise CT. Oral hygiene measures and periodontal status of school children. Int J Dent Hygiene 2011;9:143-148.

13. Al Tamami S, Peterson PE. Oral health situation of school children, mother and school teachers in Saudi Arabia. Int Dent J 1998;48(3):180-186.
14. Dawani N, Nisar N, Khan N, Syed S, Tanweer N. Oral health knowledge, attitude and self-practices of pre-school teachers of Karachi, Pakistan. J Pak Dent Assoc 2013;22(1):47-51.

15. Pradeep ST, Manish J, Anmol M, Sumanth P, Natashekara M. Knowledge, attitude and practice of dental caries and periodontal disease prevention among primary school teachers in Belgaum City, India. Pesquisa Brasileira em Odontopediatria Clinica Integrada 2011;11(1):77-83.

16. Sukhabogi JR, Shekar CBR, Hameed IA. Knowledge, attitude and practices related to oral health among English and Telugu medium school teachers in two districts of Andhra Pradesh, India: a comparative study. J Ind Assoc Public Health Dent 2014;12:306-311. 